\title{
Synchrotron X-Ray SNR Candidates Discovered in the $A S C A$ Galactic Plane Survey
}

\author{
M. Ueno ${ }^{1}$, S. Yamauchi ${ }^{2}$, A. Bamba ${ }^{3}$, H. Yamaguchi ${ }^{4}$, K. Koyama ${ }^{4}$, \\ and K. Ebisawa ${ }^{5}$ \\ ${ }^{1}$ Department of Physics, Faculty of Science, Tokyo Institute of Technology 2-12-1, \\ Oo-okayama, Meguro-ku, Tokyo 152-8551, Japan \\ email: masaru@hp.phys.titech.ac.jp \\ ${ }^{2}$ Faculty of Humanities and Social Sciences, Iwate University, 3-18-34 Ueda, Morioka, Iwate \\ 020-8550, Japan \\ ${ }^{3}$ RIKEN (The Institute of Physical and Chemical Research) 2-1, Hirosawa, Wako, Saitama \\ 351-0198, Japan \\ ${ }^{4}$ Department of Physics, Graduate School of Science, Kyoto University, Sakyo-ku, Kyoto \\ 606-8502, Japan \\ ${ }^{5}$ The Institute of Space and Astronautical Science (ISAS), Japan Aerospace Exploration \\ Agency(JAXA) 3-1-1, Yoshinodai, Sagamihara, Kanagawa 229-8510, Japan
}

\begin{abstract}
Shell-like supernova remnants (SNRs) are primary candidates for the origin of Galactic cosmic rays. However, among the known SNRs (about 220), only a small fraction has been known to exhibit the synchrotron X-ray spectrum, that is considered to be a piece of evidence for high energy particle acceleration. Synchrotron X-ray emitting SNRs are known to be systematically radio-quiet compared to the SNRs that do not emit synchrotron X-rays. Therefore, most synchrotron X-ray emitting SNR candidates may have escaped detection in the previous systematic radio surveys. On the other hand, hard X-ray surveys are effective to search for synchrotron X-ray emitting SNRs, because of its penetration power. Thus we have searched for SNRs in the $A S C A$ Galactic Plane Survey data, the first Galactic imaging survey in $>2 \mathrm{keV}$, and discovered 14 candidates. Deep follow-up observations with $A S C A, X M M$, or Chandra on 5 of them revealed 2 sources to be synchrotron X-ray emitting SNRs. Furthermore we confirmed non-thermal X-ray spectra from the other 3 sources, though the origin is yet unknown. We report the observational results and discuss the X-ray origin.
\end{abstract}

Keywords. supernova remnants; cosmic rays; Galaxy: structure.

\section{Introduction}

Since the discovery of synchrotron X-ray emission from SN1006 (Koyama et al. 1995), synchrotron X-rays and TeV $\gamma$-rays are detected in several SNRs (e.g., RX J1713.7-3946; Enomoto et al. 2002), which are evidence for high energy ( $\geqslant 10 \mathrm{TeV}$ ) electrons located in the shells of SNRs. However, synchrotron X-rays or $\mathrm{TeV} \gamma$-rays has been detected in only a small number of SNRs $(\sim 10)$ compared with the total number of SNRs now known ( $\sim 220$ excepting for filled-center type SNRs; Green 2004). Therefore, we cannot draw conclusion as to whether particle acceleration is a universal activity of SNRs or not. As a result, it is still unknown whether particle acceleration in SNRs is significant for the Galactic cosmic rays.

From the discoveries of several SNRs in the ROSAT All Sky Survey (e.g., Pfeffermann \& Aschenbach 1996), it is suggested that a part of SNRs have low radio surface brightness 
and have been escaping detection in the previous systematic radio surveys. However, due to the interstellar absorption, soft X-ray observations with $R O S A T$ are not suited for detecting sources embedded in the Galactic plane. On the other hand, hard X-rays are transparent through the Galactic plane. In addition, synchrotron X-rays are dominant against thermal emission in the hard X-ray band. Therefore, a hard X-ray survey would be the best way to find new SNRs emitting synchrotron X-rays (synchrotron X-ray SNRs) located in the Galactic plane.

On this point of view, we reanalyzed the data set of the $A S C A$ Galactic Plane Survey, which is the first imaging survey of the Galactic plane in the hard X-ray band $(>2 \mathrm{keV})$. Comprehensive studies of the survey data have been reported by Sugizaki et al. (2001). In this paper, we concentrate on new detection of unidentified diffuse hard X-ray emissions.

\section{Observations and Data Reduction}

The ASCA Galactic Plane Survey (AGPS) consists of 173 pointing observations, covering the area of the Galactic inner disk of $|l| \lesssim 45^{\circ}$ and $|b| \lesssim 0.4^{\circ}$. Since about $34 \%$ of the surveyed regions suffer from stray light from nearby bright sources, we excluded these regions in our analysis. Since GISs had 4 times larger field of views than SISs, we concentrated on the X-ray data from GIS in this SNR survey.

Exposure corrected images of the $A S C A$ Galactic Plane Survey data in the $2.0-7.0 \mathrm{keV}$ band were produced using night earth data for the background, and blank sky data for the efficiency map. We searched for unidentified extended sources in the resultant exposurecorrected 2.0-7.0 keV band images. As a result, we detected 14 new extended sources in the hard band images $(2-7 \mathrm{keV})$. Among them are 5 SNR candidates, which we have already reported (G28.6-0.1, G11.0+0.0, G25.5+0.0, G26.6-0.1, G32.45+0.1; Bamba et al. 2001; Bamba et al. 2003; Ueno et al. 2003; Yamaguchi et al. 2004), and G337.2+0.1, which was reported by Combi et al. (2005) as an SNR candidate. The hard band images of the other 8 sources are shown in Figure 1. By making radial profiles, we verified these sources are significantly extended compared with the point spread function of GIS.

\section{Discussion}

\subsection{Radio Faintness of the SNR candidates}

Radio surface brightness $(\Sigma)$ of SNRs tends to become small as their diameters $(D)$ become large, which is generally known as the $\Sigma$ - $D$ relation (Figure 2; e.g., Green 2004). Since the new SNR candidates in this paper are discovered with X-rays but not with radio observations, it is inferred that they are radio faint compared with the other SNRs. According to the $\Sigma-D$ relation, the diameters of the candidates should be large. In order to test this, the distances of the candidates are roughly estimated from the absorption column densities of the X-ray spectra, assuming the density of the ISM, $N_{\mathrm{H}}=1 \mathrm{~cm}^{-3}$. Since the $N_{\mathrm{H}}$ of two candidates is consistent with $0 \mathrm{~cm}^{-2}$ and their distances can not be determined, they are excluded here. On the other hand, the upper limit of radio brightnesses of the new SNR candidates can be estimated from the sensitivities of the previous radio surveys. The $l>0^{\circ}$ and $l<0^{\circ}$ regions are covered with the NRAO VLA Sky Survey (Condon et al. 1998) and with the Molonglo Galactic Plane Survey (Green et al. 1999), respectively. Upper limits $(3 \sigma)$ are calculated to be $6 \times 10^{-22}$ and $(1-2) \times 10^{-21} \mathrm{~W} \mathrm{~m}^{-2} \mathrm{~s}^{-1} \mathrm{~Hz}^{-1} \mathrm{sr}^{-1}$ (spectral index of 0.5 is assumed). These results are added to the $\Sigma-D$ relation in Figure 2. Although there are large uncertainties in diameters, it is seen that newly found SNR candidates are faint in radio not because they have large diameters. 

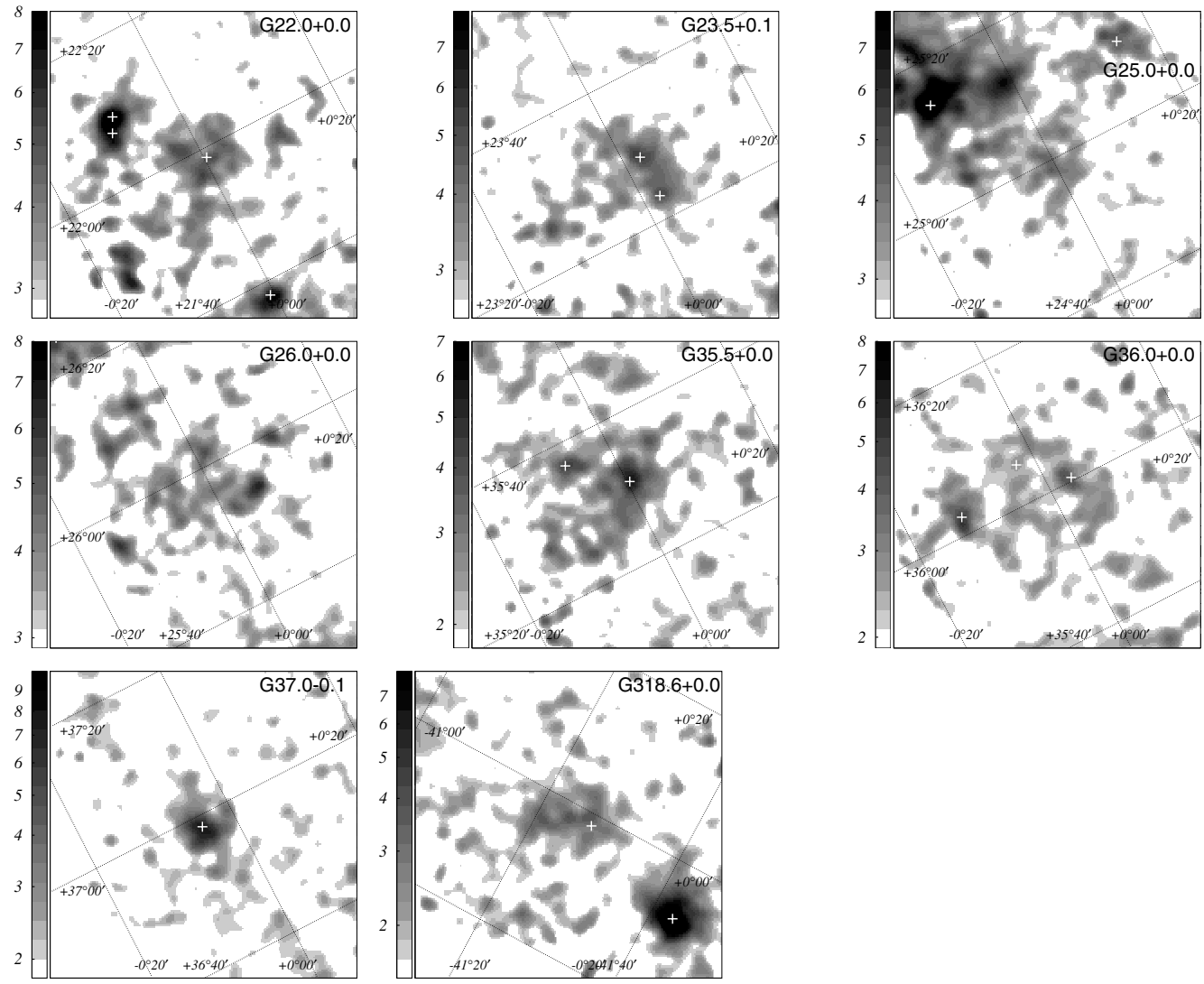

Figure 1. ASCA images of the new SNR candidates in the $2.0-7.0 \mathrm{keV}$ band. Scales are logarithmic and the numbers next to the scale bars correspond to the surface brightness $\left(\times 10^{-6}\right.$ counts $\left.\mathrm{cm}^{-2} \mathrm{~s}^{-1} \operatorname{arcmin}^{-2}\right)$. The orientation is equatorial (J2000), whereas the coordinates shown are galactic. The sources detected in Sugizaki et al. (2001) are designated with white crosses. The new SNR candidates are in the center of each image.

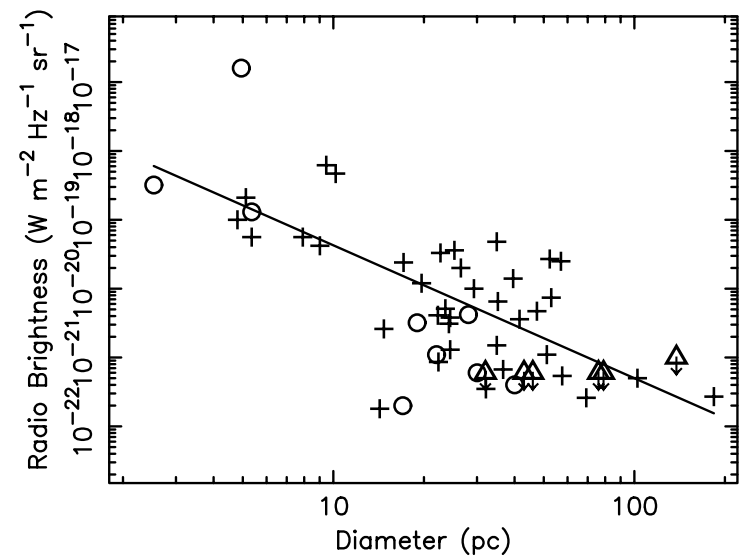

Figure 2. Radio surface brightness vs. diameter relation for shell SNRs (see Green 2004). SNR emitting synchrotron X-rays and those not are designated with circles and crosses, respectively. The new SNR candidates are shown with triangles, whose radio surface brightnesses shown are all upper limits. 
Arbutina \& Urošević (2005) suggested that SNRs located in high density media show high radio surface brightnesses, which is also theoretically deduced by Berezhko \& Völk (2004). Since synchrotron X-ray SNRs tend to be faint in radio, they may be located in low density environment.

\subsection{Expected number of synchrotron X-ray SNRs in the Galactic plane}

If we assume the diameter $(20 \mathrm{pc})$ and X-ray luminosity $\left(2 \times 10^{34} \mathrm{erg} \mathrm{s}^{-1}\right.$ in $\left.2-10 \mathrm{keV}\right)$ of SN1006 for synchrotron X-ray SNRs expected in the Galactic plane, the deepness of the $A S C A$ survey is $\sim 10 \mathrm{kpc}$ for the synchrotron X-ray SNR survey, which is limited by the angular resolution. We assume that distribution of supernova remnants is the same as that of molecular clouds. In order to estimate coverage of the $A S C A$ survey, we simply assume a finite disk model for the number density of SNRs $\eta$ as;

$$
\eta(r, z)= \begin{cases}\eta_{0} \exp \left[-\frac{1}{2}\left(\frac{|z|}{Z_{s}}\right)^{2}\right] & \left(r \leqq R_{d}\right) \\ 0 & \left(r>R_{d}\right) .\end{cases}
$$

According to Sanders et al. (1984), the scale hight of CO $(Z s)$ is $\sim 60$ pc. We assume $R_{d}=5 \mathrm{kpc}$, at which radius the Galactocentric arms are located (Sanders et al. 1984). We found that $37 \%$ of the putative SNRs are located in the region covered by the $A S C A$ survey $\left(|l|<45^{\circ},|b|<0.4^{\circ}\right.$, distance $\left.<10 \mathrm{kpc}\right)$. However, $34 \%$ of the survey region suffers from stray light and could no be used for an extended-source search. Therefore, $1 / 0.37 /(1-0.34) \sim 4$ times the number of SNRs detected in the $A S C A$ survey is expected in the Galactic plane. Since we discovered 14 candidates in the survey region and at least 2 sources, G28.6-0.1 and G32.45+0.1, are synchrotron X-ray SNRs, we expect 8 56 synchrotron X-ray SNRs in the Galactic plane.

\section{References}

Arbutina, B., \& Urošević, D. 2005, MNRAS, 360, 76

Bamba, A., Ueno, M., Koyama, K., \& Yamauchi, S. 2001, PASJ, 53, L21

Bamba, A., Ueno, M., Koyama, K., \& Yamauchi, S. 2003, ApJ, 589, 253

Berezhko, E. G., Völk, H. J. 2004, A\&A, 427, 525

Combi, J. A., Benaglia, P., Romero, G. E., \& Sugizaki, M. 2005, A\&A, 431, L9

Condon, J. J., Cotton, W. D., Greisen, E. W., Yin, Q. F., Perley, R. A., Taylor, G. B., \& Broderick, J. J. 1998, AJ, 115, 1693

Enomoto, R., et al. 2002, Nature, 416, 823

Green, A. J., Cram, L. E., Large, M. I., \& Ye, T. 1999, ApJS, 122, 207

Green, D. A. 2004, Bulletin of the Astronomical Society of India, 32, 335

Koyama, K., Petre, R., Gotthelf, E. V., Hwang, U., Matsuura, M., Ozaki, M., \& Holt, S. S. 1995, Nature, 378, 255

Pfeffermann, E., \& Aschenbach, B. 1996, Roentgenstrahlung from the Universe, 267

Sanders, D. B., Solomon, P. M., \& Scoville, N. Z. 1984, ApJ, 276, 182

Sugizaki, M., Mitsuda, K., Kaneda, H., Matsuzaki, K., Yamauchi, S., \& Koyama, K. 2001, ApJS, 134,77

Ueno, M., Bamba, A., Koyama, K., \& Ebisawa, K. 2003, ApJ, 588, 338

Yamaguchi, H., Ueno, M., Koyama, K., Bamba, A., \& Yamauchi, S. 2004, PASJ, 56, 1059 


\section{Discussion}

VINK: Wouldn't you think that the faint SNRs discovered by HESS are most likely to be $\mathrm{TeV}$ emitters because of Inverse Comptom emission from the same electrons that cause $\mathrm{X}$-ray synchrotron emission, whereas pion decay is unlikely due to the low density?

UENO: I think that's reasonable.

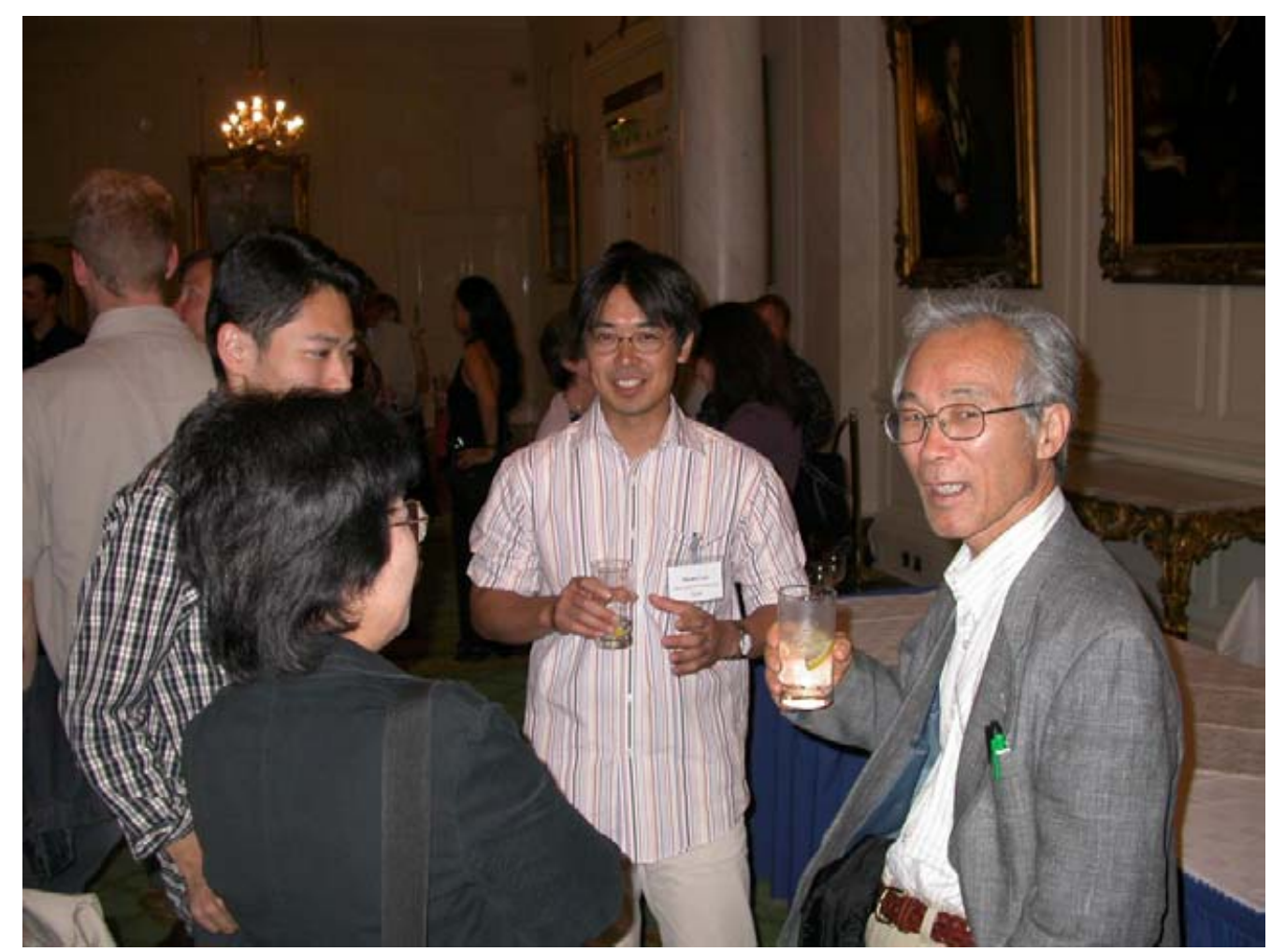

Katsuji Koyama (right) with company before the Conference Dinner; next to him Masaru Ueno. 